\title{
Experts debate-should DSM-V raise the bar for female sexual dysfunction diagnosis?
}

T

he Diagnostic and Statistic Manual of Mental Disorders (DSM) provides standard criteria for the diagnosis of mental disorders worldwide. Thus, changes to the criteria can have farreaching consequences for patients with symptoms suggestive of such conditions. The American Psychiatric Association are currently reworking the DSM fourth edition (text revision), DSM-IV-TR, with publication of DSM-V expected in May 2013.

Diagnostic criteria for female sexual dysfunction are contentious, and some specialists have suggested that the DSM-V should include revised criteria for hypoactive sexual desire disorder (HSDD) recommending that female sexual desire and female sexual arousal disorders (FSDD and FSAD, respectively) should be combined into one taxonomic category of 'sexual interest/arousal disorder (SI/AD)'. This opinion is controversial, and a US team led by Anita Clayton at the University of Virginia has recently provided evidence against the amalgamation of FSDD and FSAD in the Journal of Sexual Medicine.

Clayton et al. studied almost 500 women, 49 of whom were diagnosed with pure arousal disorder. Participants were assessed using four measures of sexual dysfunction-the Sexual Interest and Desire Inventory-Female (SIDI-F), a clinician-rated tool, and the self-rated Female Sexual Function Index, Changes in Sexual Functioning QuestionnaireFemale, and Female Sexual Distress Scale, at baseline and after 28 days. Women with FSAD scored as significantly sexually dysfunctional and sexually distressed, with fewer satisfying sexual events (SSEs) and fewer orgasms than women with normal sexual function. However, despite this evidence, most women included in the study would not meet a single criterion for SI/AD in the updated classification. The data reflect the results from the

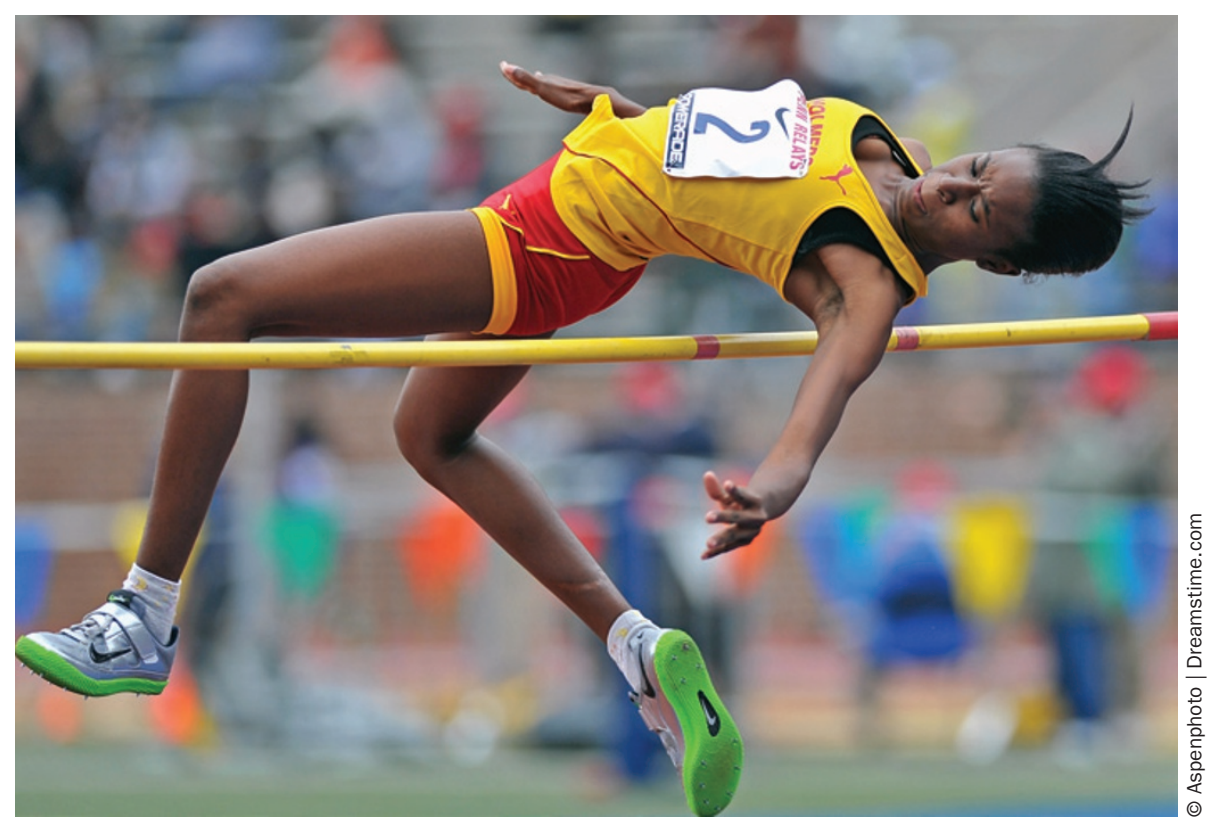

team's earlier paper focusing on women with incomplete loss of desire or sexual receptivity, in which 231 women with a previous HSDD diagnosis were compared with women with normal sexual function. Most women were rated by clinicians as meeting the criteria for diagnosis of moderate-to-marked HSDD and scored as being significantly sexually dysfunctional and sexually distressed, reporting fewer SSEs than controls, even when their HSDD was deemed mild. However, alteration of the criteria would mean that such patients would not reach the threshold for diagnosis and, presumably, treatment. The authors believe that the symptom patterns observed in these studies show that arousal disorder and desire disorder are not one and the same, and that separate diagnostic criteria are required.

The arguments on both sides are long running. Clayton's team first voiced their opinion that the bar should not be raised in 2011 (DeRogatis et al. Arch. Sex. Behav. 40, 217-219; 2011), in response to Brotto's original proposal to increase the threshold for diagnosis, but these data were criticised as being unrepresentative of women with low desire who seek treatment by Brotto and colleagues (Brotto et al. Arch. Sex. Behav. 40, 221-225; 2011). Whilst the reasons for raising the bar are reasonable-receiving a diagnosis of HSDD is not without stigma and overtreatment is a problem-Clayton et al. believe that the DSM-IV criteria are, in fact, too stringent, and that the bar should be lowered, not raised. As such, the debate is likely to continue.

\section{Annette Fenner}

\footnotetext{
Original article Clayton, A. H. et al. Intended or unintended consequences? The likely implications of raising the bar for sexual dysfunction diagnosis in the proposed DSM-V revisions: 2. For women with loss of subjective sexual arousal. J. Sex. Med. doi:10.1111/j.1743-6109.2012.02859.x

Further reading Clayton, A. H. et al. Intended or unintended consequences? The likely implications of raising the bar for sexual dysfunction diagnosis in the proposed DSM-V revisions: 1. For women with incomplete loss of desire or sexual receptivity. J. Sex. Med. 10.1111/j.17436109.2012.02850.x
} 